\title{
The role of pre-Gaia positional data in determining binary orbits with Gaia data $\dagger$
}

\author{
S. L. Ren and Y. N. Fu \\ Purple Mountain Observatory, Chinese Academy of Sciences, Nanjing, 210008, China \\ email: rensl@pmo.ac.cn or fyn@pmo.ac.cn
}

\begin{abstract}
Simulations show that incorporating the pre-Gaia positional data into the Gaia astrometric data can significantly improve the efficiency of determining binary orbits, especially for those binaries with periods from 8 to 25 years.
\end{abstract}

Keywords. stars: fundamental parameters, binaries: general, methods: data analysis

\section{Introduction}

In recent papers, simulations revealed that, even with their unprecedented precision, Gaia astrometric data can only be used to reliably measure the orbits of binaries with periods less than 6 years (Lattanzi et al., 2004). But for those binaries with periods larger than 6 years, the reliability of orbital measurements becomes very low because the observational time span is shorter than the orbital period during Gaia mission. In other words, in order to obtain reliable orbit solutions of these binaries, one has to incorporate the other data based on long-term observations (pre-Gaia data). There are two kinds of pre-Gaia data, namely radial velocity data and positional data. The former one is believed to be useful as it can be inferred from similar situations of Hipparcos binaries (e.g., Jancart et al., 2005). The role of latter one is discussed in this paper. Since 1990, the precision of the pre-Gaia positional data has already reached about several millarcseconds, but there are only a few data available for some binaries. Therefore, these data themselves aren't sufficient to be used to derive reliable orbits of binaries. This is the case for some binaries in the fourth catalog of interferometric measurements of binary stars (Hartkopf et al., 2001). Compared to Gaia astrometric data, these data have the advantage of long time span. It is then interesting to know whether these data of the long observational time span can play a role in determining binary orbits after Gaia mission? In order to answer this question, we do the following simulations.

\section{Simulations}

First, one hundred simulated orbits are produced with periods randomly distributed in the range from 6 to 25 years, relative semi-major axis chosen as 50 mas (higher than resolution limits of many large telescopes), astrometric semi-major axis chosen as 1 mas (corresponds to Gaia data with signal-to-noise ratio about 100/1), and other parameters randomly distributed in their respective ranges.

Then, similar to Lattanzi et al., (2000), Gaia astrometric data are simulated with precision 0.01 mas, and data number 60 on average. Pre-Gaia positional data are simulated

$\dagger$ Supported by the National Natural Science Foundation of China (Grant Nos. 10473025 and 10703014) 

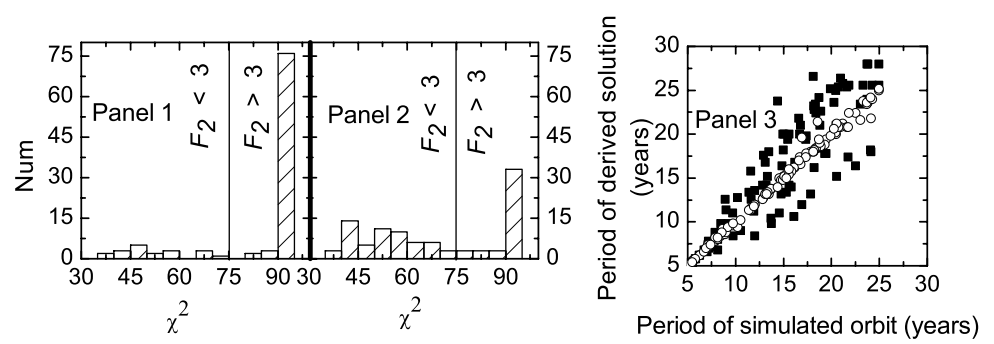

Figure 1. Panel 1: the distribution of $\chi^{2}$ of the solutions derived from Gaia data only; panel 2: the distribution of $\chi^{2}$ of the solutions from combining Gaia and pre-Gaia data; panel 3: periods of simulated orbits versus derived solutions. The filled squares indicate orbit solutions from Gaia data only, and the empty circles indicate the solutions from combining Gaia and pre-Gaia data.

with precision 5 mas, data number 5 , and observational epochs randomly distributed in the range from 1990 to 2010 years.

By only fitting Gaia data and fitting both Gaia and pre-Gaia data, two kinds of orbit solutions can be derived for each binary.

\section{Results}

In order to decide the acceptability of a derived orbit solution, we use the $F_{2}$ indicator (e.g., Lattanzi et al., 2004). If $\left|F_{2}\right|<3$, the derived solution is acceptable. More than $60 \%$ of derived solutions from both Gaia and pre-Gaia data are acceptable, the percentage is reduced to $15 \%$ for the solutions derived from Gaia data only. The distributions of $\chi^{2}$ are shown for the two different kinds of solutions in figure 1, respectively.

We compare the periods of derived solutions with that of simulation orbits in panel 3. If period $<8$ years, solutions are all in accordance with that of simulation orbits, if period $>8$ years, solutions derived from combining Gaia and pre-Gaia data are still in good agreement with that of simulation orbits, which is not the case for the solutions from Gaia data only.

\section{Conclusion}

Above simulations show that pre-Gaia positional data can significantly improve the efficiency of determining orbits of binaries, especially for those with periods $>8$ years.

\section{Acknowledgements}

Thanks prof. W. J. Jin for her useful suggestions.

\section{References}

Hartkopf, W. I., McAlister, H. A. \& Mason, B. D. 2001, AJ 122, 3480

Jancart, S., Jorissen, A., Babusiaux, C. \& Pourbaix, D. 2005, A $\& A 4442,365$

Lattanzi, M. G., Spagna, A., Sozzetti, A. \& Casertano, S. 2000, MNRAS 317, 211

Lattanzi, M. G., Jancart, S., Morbidelli, et al. in C. Turon, K. S. O'Flaherty, M. A. C. Perryman eds., 2005 Proceedings of the Gaia Symposium "Detection and Characterization of Extrasolar Planets with Gaia", P. 251 